\title{
Evidence that ebolaviruses and cuevaviruses have been diverging from marburgviruses since the Miocene.
}

An understanding of the timescale of evolution is critical for comparative virology but remains elusive for many RNA viruses. Age estimates based on mutation rates can severely underestimate divergences for ancient viral genes that are evolving under strong purifying selection. Paleoviral dating, however, can provide minimum age estimates for ancient divergence, but few orthologous paleoviruses are known within clades of extant viruses. For example, ebolaviruses and marburgviruses are wellstudied mammalian pathogens, but their comparative biology is difficult to interpret because the existing estimates of divergence are controversial. Here we provide evidence that paleoviral elements of two genes (ebolavirus-like VP35 and NP) in cricetid rodent genomes originated after the divergence of ebolaviruses and cuevaviruses from marburgviruses. We provide evidence of orthology by identifying common paleoviral insertion sites among the rodent genomes. Our findings indicate that ebolaviruses and cuevaviruses have been diverging from marburgviruses since the early Miocene. 
1 Evidence that ebolaviruses and cuevaviruses have been diverging from marburgviruses

2 since the Miocene.

3 Derek J. Taylor ${ }^{1}$, Matthew J. Ballinger, Jack J. Zhan, Laura E. Hanzly, and Jeremy A. Bruenn

4 Department of Biological Sciences, The State University of New York at Buffalo, Buffalo, NY 5 14260, USA.

$6 \quad{ }^{1}$ Correspondence and requests for materials should be addressed to D. J. T. (e-mail:

7 djtaylor@buffalo.edu). 


\section{INTRODUCTION}

Knowledge of the timescale of evolution is a critical part of understanding host-virus

10 interactions. Studies of viral systems that have evolved for tens of millions of years would

11 perhaps be complicated by host shifts, broad geographic distributions, and functional

12 divergences. Knowledge of divergence times might also affect design of vaccines and programs

13 that identify emerging pathogens. However, the timescale of viral evolution has remained

14 controversial (Gilbert \& Feschotte, 2010; Holmes, 2003;Patel, Emerman \& Malik, 2011; Sharp

$15 \&$ Simmonds, 2011; Wertheim \& Kosakovsky Pond, 2011). Fossil and geographic calibrations are

16 normally absent and evolutionary rates based on isolation dates of historical strains often grossly

17 underestimate long-term divergences. Part of the underestimation is due to the failure of

18 commonly used models to accommodate the strong purifying selection of viral proteins

19 (Duchêne, Holmes \& Ho 2014; Patel, Emerman \& Malik, 2011; Wertheim \& Kosakovsky Pond,

20 2011). But other aspects of viruses such as variation in replication rate also affect clock-based

21 estimates (Hicks \& Duffy, 2014; Holmes, 2003). Even models that accommodate purifying

22 selection will eventually encounter a mutational saturation problem (Wertheim \& Kosakovsky

23 Pond, 2011). Age estimation using co-phylogeny with the host seems more promising, but

24 detailed co-phylogenies are still uncommon and can be complicated by host jumping (Holmes,

25 2003).

26 Another potentially reliable source of minimum divergence times is endogenous

27 paleoviral elements (Katzourakis et al., 2007). Over the last decade, evidence of the most

28 unexpected class of paleoviral elements, Non-retroviral Endogenous RNA Viral Elements

29 (NERVE's), has been provided for each major eukaryotic group by sequencing across the

30 integration boundaries of putative viral elements and host genomes (Crochu et al., 2004; Horie et

31 al., 2010; Liu et al., 2010; Tanne \& Sela, 2005; Taylor \& Bruenn, 2009; Taylor, Leach \& 
32 Bruenn, 2010). BLAST searches of animal genome databases alone suggest that representatives

33 of all known viral genome architectures are involved in the formation of paleoviral elements

34 (Belyi, Levine \& Skalka, 2010; Katzourakis \& Gifford, 2010). Agreement of the NERVE

35 phylogeny with the host phylogeny is evidence of insertion in the genome of a common ancestor.

36 This pattern can be complicated by the formation of non-orthologous copies from independent

37 insertions, duplications and horizontal transfers (Taylor \& Bruenn, 2009). But, these

38 complications become less important in phylogenies with greater taxonomic representation. Even

39 stronger support for orthology is provided by evidence of common integration sites for NERVE's

40 (Katzourakis \& Gifford, 2010; Taylor, Leach \& Bruenn, 2010). If the host genomic flanking

41 sequences show significant similarity (microsynteny), then it is unlikely that NERVE insertions

42 are independent (given the large number of possible insertions sites in eukaryotic genomes).

43 Ballinger et al. (2014), for example were able to identify microsyntenous NERVE's from a novel

44 bunyavirid for the genus Drosophila and estimate a minimum date of $42 \mathrm{MY}$. As with the hosts,

45 the paleoviral sequences are "sister" phylogenetic groups supporting a single origin. So, the

46 strongest paleoviral calibrations satisfy two conditions: evidence of a common integration site in

47 the host genomes and of a similar phylogeny of host and paleovirus.

Age estimation by synteny has limitations. As with real fossils, the age estimates based on

49 paleoviruses are minimum dates based on the material currently available. The synteny of the

50 oldest copies may be difficult to establish because of chromosomal evolution that occurred post-

51 integration. An additional source of uncertainty arises from the dating of actual host fossils and of

52 host divergences. It also may be imagined that NERVE-virus phylogenetic comparisons suffer

53 because the mutation rate of RNA viruses is orders of magnitude greater than the mutation rate of

54 the hosts. However, this reasoning ignores the growing evidence of strong purifying selection in 
55 viruses -- a high mutation rate is not necessarily reflected in the amino acid substitution rate.

56 Indeed, ancient NERVE's would simply be undetectable for viruses that diverged rapidly at the

57 amino acid level.

58 Still, very few paleoviral calibrations are available for internal nodes of phylogenies of

59 extant RNA viruses. Taylor et al. (2011) reported that the family Filoviridae must be at least 13

60 MY old because fossil copies of the NP and VP35 -like genes have been integrated at common

61 sites shared among the mouse (Mus musculus) and the rat (Rattus norvegicus) NCBI reference

62 genomes. However, paleoviral calibrations that would permit estimation of a minimum

63 divergence date for extant ebolaviruses, cuevaviruses and marburgviruses are unknown.

64 Molecular estimates of the age of the common ancestor of extant known filovirids fall into two

65 time ranges. One range is coincident with the rise of agriculture in humans from 7,100-10400

66 years ago (Carroll et al., 2013; Suzuki \& Gojobori, 1997). The other range is from the Middle

67 Pleistocene at 155,000 years ago (Negredo et al., 2011). Still others have stated that the oldest

68 extant filovirids (and other RNA viruses) are in a divergence zone that is simply recalcitrant to

69 molecular clock dating (Wertheim \& Kosakovsky Pond, 2011). In such a zone, even models that

70 have been corrected for purifying selection will fail to glean temporal signal that has been

71 destroyed by synonymous substitutions.

72 Here we show that the limitations for using the molecular clock to date RNA viruses can

73 be mitigated by the discovery and dating of orthologous paleoviral elements within clades of

74 extant RNA viruses. From congruent evidence of two genes we report that the divergence of the

75 known extant filovirids (marburgviruses, ebolaviruses, and cuevaviruses) is likely older than the

76 Miocene ancestor of the hamsters and voles - a separation that is orders of magnitude greater

77 than previous Holocene and Middle Pleistocene estimates of divergence. 


\section{MATERIALS \& METHODS \\ PCR and DNA Sequencing}

We obtained a tail clipping of a dead specimen of a meadow vole from western New York state. We extracted DNA using the Epicentre Quickextract kit. PCR reactions with primers based on the genome assembly of Microtus ochrogaster (Wagner, 1842) were designed to amplify from the exon across the VP35-like gene insert boundary and from the intergenic region across the NPlike boundary of putative orthologs of hamster loci. Primers based on the assembly of the genome of the prairie vole (Microtus ochrogaster) for the VP-35-like region were:

GAGCAGGCTTTTGCTTTGATTCCAG(forward), CTGATCTCAGCTATCTCACCTGCTAAGA(reverse). For the NP-like region primers were: TGCATTGCTTGGCCGTTCTGTATGC(forward) and ATAAGACATGCTCCTTGTCTTGAAG(reverse). The 5' end of the mitochondrial COI gene region was also PCR amplified using custom primers based on published sequences of Microtus: TTACAGTCTAATGCTTTACTCAGCC (forward), ACTTCTGGGTGTCCGAAGAATCAG (reverse). PCR products were purified and submitted for Sanger sequencing at the Roswell Park Cancer Institute's Biopolymer facility. Chromatograms were assembled and trimmed using Geneious 7.0 (Biomatters).

\section{Bioinformatics}

Genomic sequences from the NCBI WGS and reference genome databases were obtained by using protein sequences of NP and VP35 of Ebola virus as queries. We used the tBLASTn algorithm with mammals as a taxonomic delimiter. Resulting contigs with an expect value $<10^{-5}$ were retained and exported. The NP and VP35 protein sequences from Ebola virus were then used to search for significant matches of the contigs using the FASTA program tfasty (Pearson, 
101 2004). Translated sequences were then prepared as a FASTA format alignment by changing the

102 NCBI header to a user-friendly name with a Python script. Sequences were submitted to the E-

103 INS-I algorithm of MAFFT for multiple sequence alignment (Katoh \& Standley, 2014). The

104 resulting alignment file was then submitted to the transitive consistency score (TCS) algorithm of

105 T-Coffee to assess alignment reliability (Chang, Di Tommaso \& Notredame, 2014). Unfiltered

106 and filtered alignment files in FASTA format for each filovirid-like gene are provided in the

107 Supplemental information. The lowest scoring categories of columns were successively filtered

108 from the alignments using a Python script to assess the effect of rapidly evolving or differently

109 evolving sites on branch support. To assess possible effects of increased rate evolution at the tips

110 of the tree, the sequences of ancestral nodes of endogenous viral clades were estimated using the

111 three ancestral reconstruction methods (Delport et al., 2010) in HyPhy (joint maximum

112 likelihood, marginal maximum likelihood, and mode of the posterior distribution of characters)

113 with a JTT + gamma substitution model. Midpoint and outgroup rooting was carried out in

114 Figtree 1.4, with the outgroup being clades of mammalian filovirid-like NERVE's outside of the

115 clade of extant known filovirids. Seaview 4.5.2 (Gouy, Guindon \& Gascuel, 2010) was further

116 used explore rooting the tree "at the point in the tree that minimizes the variance of root-to-tip

117 distances". Protein models were fit to the resulting alignments using Partitionfinder protein

118 (Lanfear et al., 2012). Phylograms were estimated using Bayesian MCMC using MrBayes 3.2.2

119 (Ronquist et al., 2012) as implemented at the CIPRES science gateway (Miller, Pfeiffer \&

120 Schwartz, 2010). Priors for MrBayes included the amino acid model fixed as JTT (Jones). The

121 sampling frequency was every 1000 generations with the MCMC analysis continuing until the

122 average standard deviation of split frequencies was less than 0.01 . We used a burnin fraction of

$123 \quad 0.25$ and a random starting tree. Branch reliability was assessed with Bayesian posterior 
124 probability values and by approximate likelihood ratio tests (aLRT). Maximum likelihood was

125 carried out in PhyML 3.1 (Guindon et al., 2009) as implemented in Seaview 4.5.2 with the

126 subtree pruning and regrafting search algorithm (SPR). Phylograms were visualized in Figtree 4.1

127 (Rambaut, 2012) and Adobe Illustrator.

128 Microsynteny of NERVE insertion sites was assessed by carrying out a BLAST search

129 with NERVE's as the queries and the annotated reference genomes of rodents as the databases.

130 NERVE-containing segments were compared among rodents after using the progressive

131 alignment algorithm in the Mauve (Darling, Mau \& Perna, 2010) plugin of Geneious 7

132 (Biomatters). Patristic genetic distances (measured from branches on a gene tree) based on

133 nucleotide alignments of filovirid-like regions in rodents and their extracted intronic or intergenic

134 backgrounds were estimated in Seaview 4.5.2 (Gouy, Guindon \& Gascuel, 2010) using the HKY

135 distance (Hasegawa, Kishino \& Yano, 1985) with among site rate variation being optimized in

136 four categories.

\section{RESULTS AND DISCUSSION}

138 Significant Blast expect values were found for 50 NP-like sequences from mammalian genomes

139 and 11 VP35-like sequences. Only one assembly per species was retained for the analysis. We

140 detected several previously unknown filovirid-like NERVE's from rodent genomes. These

141 included NERVE's from the Upper Galilee Mountains blind mole rat (Spalax galili), the golden

142 hamster (Mesocricetus auratus), the prairie vole (Microtus ochrogaster), and the North American

143 Deermouse (Peromyscus maniculatus bairdii). An additional NERVE sequence was amplified by

144 PCR from the meadow vole (Microtus pennsylvanicus). Our cytochrome c oxidase subunit 1

145 mitochondrial sequence is consistent with the taxonomic identification as it yielded a $99 \%$

146 identity score with sequences from meadow voles (e.g., JQ350481.1). Additional known 
147 filovirid-like sequences from mouse and rat genomes and those present in EST libraries or

148 isolated from mammalian genomes by PCR (Taylor, Leach \& Bruenn, 2010; Taylor et al., 2011)

149 were excluded because we focused on the relationships within the clade of known extant

150 filovirids.

151 Both the NP-like (Fig. 1) and the VP35-like (Fig. 2) sequence phylogenies revealed a

152 clade of cricetid rodent sequences within the clade of extant filovirids. Indeed, both genes had

153 cricetid clades paired with ebolaviruses and cuevaviruses to the exclusion of marburgviruses.

154 Taylor et al. (Taylor et al., 2011) had previously identified this position for the genomes of a

155 single rodent, the striped dwarf hamster (Cricetulus barabensis griseus), but here we have found

156 support for sequences of other cricetid rodents forming a monophyletic clade. This is the most

157 closely related clade of endogenous mammalian genes known for filovirids. The phylogenetic

158 positions are strongly supported by posterior probabilities and aLRT's.

The occurrences are unlikely to be assembly artifacts because the genomes in question are NCBI reference genomes with strong sequence coverage. The striped dwarf hamster (C. griseus) has independent genome assemblies that agree on the insert locations. Also, the only mammalian species in this filovirid clade are cricetid rodents, some of which have identical insertion

163 locations in their genomes. The pattern of shared insertion among monophyletic taxa is a

164 prediction of common ancestry rather than of assembly artifacts. Finally, we carried out PCR in

165 the meadow vole using primers designed to flank the VP35-like region of the prairie vole (which

166 has a genome project). The PCR reaction was positive and the sequence had strong identity to the 167 microtine sequence from the genome assembly (Fig. 3). Excluding indels the sequence across this 168 putative insert region showed 94\% (583 nt) identity between the genome assembly of the pairie 169 vole (M. ochrogaster) and the PCR product of the meadow vole (M. pennsylvanicus). Our partial 170 sequence is consistent with an orthologous insert of a VP35-like sequence in the genome of the 171 meadow vole (M. pennsylvanicus) and confirms the assembled location of this region in rodents 172 in the 3 ' intron of the Taxl-binding protein 1 (TAX1BP1) gene locus. 
We explored the possibility of systematic error contributing to the pairing of cricetid

174 sequences with ebolaviruses. Long branch attraction (LBA) can occur with real data even under

175 model-based approaches that account for among-site rate variation (Anderson \& Swofford, 2004;

176 Omilian \& Taylor, 2001; Taylor \& Piel, 2004). In some cases, distant outgroups can play a role

177 in LBA (Sanderson et al., 2000). It is expected that support for LBA groupings will be reduced if

178 sites that are rapidly evolving or that lack agreement among pairwise alignments are reduced in

179 the data. However, with the VP35-like and NP-like genes, successively filtering such sites

180 according to the transitive consistency score either increased support for the observed

181 cricetid/ebolavirus pairing or had no effect on support (Fig. 4). Support for the internal position

182 eroded only when the number of sites had been reduced to less than $15 \%$ of the data for the NP

183 gene and 34\% for the VP35 gene. We also note that similar LBA conditions between the genes

184 are lacking because the branch length patterns are reversed for the two genes. For the VP35

185 phylogeny, marburgviruses have the longest distance to the root among extant viruses, but for the

186 NP gene, ebolaviruses have the longest distance to the root. Yet, in each case the cricetid

187 sequences group with ebolaviruses and cuevaviruses. The increase in support for this clade with

188 filtering, then, is most likely a result of culling evolutionary noise from the mammalian genes.

189 Most of the NERVE's are pseudogenes that accumulate evolutionary noise in the form of indels

190 and reading frame disruptions.

To further explore a role for outgroups in affecting the relationships of the ingroup we

192 carried out several analyses with new alignments that omitted outgroup taxa. Every analysis for

193 the VP35-like gene alignment grouped the cricetid sequences inside the clade of extant filovirids

194 with strong support (Table 1). The ingroup analysis using the complete NP-like alignment

195 indicated that cricetid sequences grouped outside of the extant filovirids. However, replacing the

196 cricetid clade with ancestral reconstructions of this clade of pseudogenes moved the cricetid NP-

197 like sequences internal to the extant filovirids. Ancestral reconstruction of the NERVE clade

198 reduced gaps that may have contributed to a biased attraction of extant viral genera with fewer

199 indels. In support of this notion, removing the most rapidly evolving sites and using ancestral 
reconstructions of the cricetid copies gave the same results as the outgroup-rooted sequences for

201 both genes. So, phylogenetic analysis of ingroup sequences alone after reducing the most rapidly

202 evolving and rapidly eroding sites (including indels) indicates that the position of the cricetid

203 sequences within extant filovirids is unlikely to be the result of an outgroup bias.

Microsynteny was observed among a monophyletic clade of VP35-like NERVE's in cricetid rodents. The (Microtus, (Cricetulus, Mesocricetus)) grouping agrees with rodent taxonomy, with the microsynteny being apparent at the genic and the nucleotide level. Namely, these cricetid rodents share a VP35-like insert in the 3'-most intron of the Tax1-binding protein 1 (TAX1BP1) gene locus (Fig. 5). This insertion site is also identical to that of the partial VP35-like gene from the meadow vole (M. pennsylvanicus) that we amplified by PCR. TAX1BP1 is involved in the down regulation of inflammation genes (Verstrepen et al., 2011). Interestingly,

211 both the TAX1BP1 of mammals (Parvatiyar, Barber \& Harhaj, 2010) and VP35 of ebolaviruses

212 (Basler et al., 2003; Hartman, Towner \& Nichol, 2004; Hartman et al., 2008) inhibit IRF

213 (interferon regulatory factor)3, a critical transcription factor for the initiation of viral innate

214 immunity in mammals. The host's inhibition of the interferon pathway is necessary to prevent the

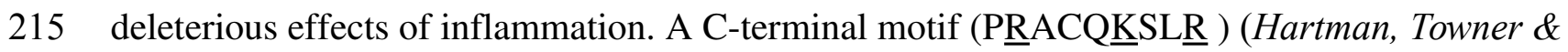

216 Nichol, 2004; Hartman et al., 2008) of theVP35 of ebolaviruses targets the same transcription

217 factor to inhibit mammalian innate immunity to viruses. Unlike the more divergent VP35-like

218 NERVE's from bats (Belyi, Levine \& Skalka, 2010), the cricetid VP35-like NERVE's show

219 evidence of conservation of the three basic residues affecting the interferon response.

220 Specifically, two of the striped dwarf hamster hamster (C. griseus) NERVE's and one of the

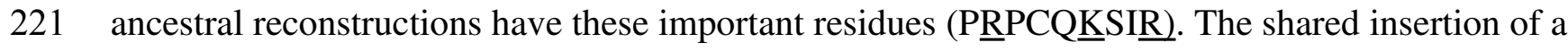

222 viral gene that inhibits IFR3 in a mammalian gene that plays a key role in the inhibition of IFR3

223 immediately suggests selective maintenance of the integration. The VP35 of Ebola virus fails to

224 inhibit the type I interferon response in hamsters (Ebihara et al., 2013). Also, Ebola virus

225 infections in hamsters cause downregulation of proinflammatory cytokines, while still inducing a

226 type I interferon response (Ebihara et al., 2013). Although intron sequence variation is known to 
227 be functional in mammals (Praetorius et al., 2013), we are unaware of functional studies of the 228 VP35-interrupted TAX1BP1 of rodents. Functional studies are needed to address the possibility 229 that co-option of a viral interferon pathway regulator (VP35) contributes to the immune response 230 of hamsters.

Microsynteny was also observed for the NP-like genes of cricetid rodents (Fig. 6). The 232 oldest case of microsynteny involved inserts in the same intergenic region between the gliomedin 233 and cytochrome P450 19A1-like loci shared by rodents of the following genera: Microtus, 234 Mesocricetus, and Cricetulus. Hamsters of the genera Cricetulus and Mesocricetus had very high 235 similarity of flanking sequences (Fig. 6) indicating a shared insert site. The insert in the vole genome was just under $10 \mathrm{kbp}$ from the hamster insert site. Possible causes of the differing insert

237 location could be a modest rearrangement, an assembly artifact, an independent insert in the same 238 intergenic region, or tandem duplication followed by loss of the original insert. We sequenced 239 across the putative insert boundary using DNA from the meadow vole (M. pennsylvanicus) as a 240 template to assess the assembly. The assembly and location of the insert was verified by the 241 sequence, which had at least $91 \%$ nucleotide sequence identity with the assembly of the pairie 242 vole (M. ochrogaster). We then compared the pairwise sequence divergences of putatively 243 orthologous NERVE's, under the expectation that orthologous NERVE's would evolve at a 244 similar or slower rate than the background intron or intergenic region of their insertion. The NP-

245 like insert of the vole evolved at about twice the rates of the intronic, intergenic and other

246 NERVE comparisons, suggesting that the vole insert may not be orthologous to the hamster insert 247 (Fig. 7). A dotplot comparison of this sequence revealed no evidence for recombination. We 248 conclude that there is strong evidence for the orthology of NP-like inserts for hamsters of the 249 genera Cricetulus and Mesocricetus in the intergenic region between gliomedin and cytochrome 250 P450 19A1-like loci. However, the NP-like insert of the vole may be an independent insert or a 251 paralog. If true, then gene order evidence alone for mammals may be too crude a measure to 252 evaluate orthology for NERVE's. 
The combination of monophyly, phylogenetic agreement with rodent subfamilies,

254 topological agreement between genes, and microsynteny indicate that the cricetid rodent VP35-

255 like insertion was present in the common ancestor of hamsters and voles and the NP-like

256 insertion was present in the common ancestor of hamsters of the genera Cricetulus and

257 Mesocricetus. It may be argued that integrated viruses evolve by a different mode than do extant

258 viruses or that integrated viruses are too distant from extant viruses to be biologically relevant.

259 However, strong purifying selection has been demonstrated to occur in both RNA viruses

260 (Wertheim \& Kosakovsky Pond, 2011) and in their integrated eukaryotic versions (Taylor et al.,

261 2011). Some of these show both RNA expression products and purifying selection (Ballinger et

262 al., 2014). In at least one case an integrated RNA viral gene produces a protein product (Taylor et

263 al., 2013). Under strong purifying selection, similarity at the amino acid level can be preserved

264 despite differences in the mutation rates for many millions of years. Our analysis of the transient

265 consistency scores indicates that if differing modes of evolution exist, they have little effect on

266 the major relationships of the sequences from inferred amino acids. Given our evidence for

267 orthology of the ebolavirus-like genes, we can provide a minimum estimate of the age of the

268 insert as the age of the common ancestor of hamsters and voles. Molecular clock estimates using

269 fossil calibrations agree that hamsters and voles had a common ancestor in the Miocene

270 (Abramson et al., 2009; Fabre et al., 2012; Horn et al., 2011; Jansa, Barker \& Heaney, 2006;

271 Parada et al., 2013; Steppan, Adkins \& Anderson, 2004). Indeed, these studies indicate a

272 divergence date of about $18 \mathrm{MY}$ ago (error bars span much of the Miocene). The common

273 ancestor of hamsters of the genera Cricetulus and Mesocricetus has also been estimated in the

274 Miocene at 7-12 MY ago (Neumann et al., 2006). If the phylogenetic placement of the cricetid

275 NERVE's within known extant filovirids is correct, then the divergence of marburgviruses from

276 other filovirids (ebolaviruses and cuevaviruses) must also be at least as old as the Miocene. This

277 age is orders of magnitude older than previously thought and likely will aid in understanding the

278 comparative biology of filovirids. The differing genome architecture, transcriptional editing, and

279 immunological reactivity of cuevavirions, ebolavirions, and marburgvirions (Kuhn et al., 2010)

280 had a much longer time to evolve than the rise of agriculture. Our results provide strong evidence 
281 that molecular clock based estimates for extant filovirids have been severely underestimated as

282 predicted by the saturation problem posed by Wertheim et al. (Wertheim \& Kosakovsky Pond,

283 2011). However, our methods also provide a solution to this problem - dating of orthologous

284 paleoviruses.

Small rodents appear overrepresented amongst the NERVE's of filovirids. The analysis

286 here further bolsters this pattern with the mouse-related clade being represented in each of the

287 three deep clades of filovirids (fossil and extant). Indeed, the genome of the North American

288 deermouse (P. maniculatus bairdii) has NERVE's from all three major filovirid clades,

289 suggesting multiple historical integrations of divergent filoviral lineages. It is unknown why the

290 genomes of mouse-like rodents appear overrepresented in the list of mammals with filovirid-like

291 NERVE's. Because NERVE's originate as rare macromutations, nearly all inserts will be lost by

292 failing to achieve integration into germ-line cells. Those that become endogenous will most likely

293 disappear after genetic drift or selection. Casual infection alone, then, is unlikely to result in the

294 fixation of numerous long-lived integrations in rodents (Johnson, 2010). The evidence of

295 repeated genomic and evolutionary interactions of filovirids (including the extant clade of

296 filovirids) with cricetid rodents should be considered when comparing the differing

297 immunological responses among mammals to infections with modern filovirids (Wahl-Jensen et

298 al., 2012).

Cricetid rodents have captured orthologous NP and VP35-like gene segments from

300 filovirids that group phylogenetically within the extant filovirids. The sharing of these genomic

301 sections provides the first evidence that extant known filovirids have been diverging since the

302 Miocene. The results show that fossil copies of RNA viruses can provide minimum estimates of

303 divergence in a divergence range that is recalcitrant to present molecular clock methods with

304 extant viruses. Our results also bolster evidence that mouse-like rodents have had repeated

305 genomic interactions with filovirids. Our finding of a filoviral insert that interrupts an important 
306 regulator of the innate antiviral response also informs hypotheses regarding the possible 307 biological significance to the mammalian host of such inserts. 
308 ACKNOWLEDGEMENTS

309 We thank Dr. Solon Morse for the tail clipping of the meadow vole. 
311 Abramson I, Lebedev S, Tesakov S, Bannikova A. 2009. Supraspecies relationships in the subfamily Arvicolinae (Rodentia, Cricetidae): An unexpected result of nuclear gene analysis. Molecular Biology 43:834-846.

Anderson FE, Swofford DL. 2004. Should we be worried about long-branch attraction in real data sets? Investigations using metazoan 18S rDNA. Molecular Phylogenetics and Evolution 33:440-451.

Ballinger MJ, Bruenn JA, Hay J, Czechowski D, Taylor DJ. 2014. Discovery and evolution of

Belyi VA, Levine AJ, Skalka AM. 2010. Unexpected inheritance: multiple integrations of ancient bornavirus and ebolavirus/marburgvirus sequences in vertebrate genomes. PLoS Pathogens 6:e1001030.

\section{Carroll SA, Towner JS, Sealy TK, McMullan LK, Khristova ML, Burt FJ, Swanepoel R,} Rollin PE, Nichol ST. 2013. Molecular Evolution of Viruses of the Family Filoviridae Based on 97 Whole-Genome Sequences. Journal of Virology 87:2608-2616.

Chang J-M, Di Tommaso P, Notredame C. 2014. TCS: A new multiple sequence alignment reliability measure to estimate alignment accuracy and improve phylogenetic tree reconstruction. Molecular Biology and Evolution 31: 1625-1637. Micco P, de Lamballerie X. 2004. Sequences of flavivirus-related RNA viruses persist in DNA form integrated in the genome of Aedes spp. mosquitoes. Journal of General 
Virology 85:1971-1980.

336 Darling AE, Mau B, Perna NT. 2010. progressiveMauve: multiple genome alignment with gene 337 gain, loss and rearrangement. PloS one 5:e11147.

338 Delport W, Poon AF, Frost SD, Pond SLK. 2010. Datamonkey 2010: a suite of phylogenetic 339 analysis tools for evolutionary biology. Bioinformatics 26:2455-2457.

340 Duchêne S, Holmes EC, Ho SY. 2014. Analyses of evolutionary dynamics in viruses are hindered by a time-dependent bias in rate estimates. Proceedings of the Royal Society B: Biological Sciences 281:20140732

Ebihara H, Zivcec M, Gardner D, Falzarano D, LaCasse R, Rosenke R, Long D, Haddock E, Fischer E, Kawaoka Y, Feldmann H. 2013. A Syrian Golden Hamster Model Recapitulating Ebola Hemorrhagic Fever. Journal of Infectious Diseases 207:306-318.

Fabre PH, Hautier L, Dimitrov D, Douzery EJ. 2012. A glimpse on the pattern of rodent diversification: a phylogenetic approach. BMC Evolutionary Biology 12:88.

Gilbert C, Feschotte C. 2010. Genomic fossils calibrate the long-term evolution of hepadnaviruses. PLoS biology 8:e1000495

Gouy M, Guindon S, Gascuel O. 2010. SeaView version 4: a multiplatform graphical user interface for sequence alignment and phylogenetic tree building. Molecular Biology and

Guindon S, Delsuc F, Dufayard J-F, Gascuel O. 2009. Estimating maximum likelihood phylogenies with PhyML. Springer. Evolution 27:221-224.

Hartman AL, Towner JS, Nichol ST. 2004. A C-terminal basic amino acid motif of Zaire ebolavirus VP35 is essential for type I interferon antagonism and displays high identity 
with the RNA-binding domain of another interferon antagonist, the NS1 protein of influenza A virus. Virology 328:177-84.

362 Hasegawa M, Kishino H, Yano T-A. 1985. Dating of the human-ape splitting by a molecular clock of mitochondrial DNA. Journal of molecular evolution 22:160-174.

364 Hicks AL, Duffy S. 2014. Cell tropism predicts long-term nucleotide substitution rates of mammalian RNA viruses. PLoS pathogens 10:e1003838

Holmes EC. 2003. Molecular clocks and the puzzle of RNA virus origins. Journal of Virology 77:3893-3897.

\section{Horie M, Honda T, Suzuki Y, Kobayashi Y, Daito T, Oshida T, Ikuta K, Jern P, Gojobori T,} Coffin JM. 2010. Endogenous non-retroviral RNA virus elements in mammalian genomes. Nature 463:84-87.

Horn S, Durka W, Wolf R, Ermala A, Stubbe A, Stubbe M, Hofreiter M. 2011. Mitochondrial genomes reveal slow rates of molecular evolution and the timing of speciation in beavers (Castor), one of the largest rodent species. PloS one 6:e14622.

Jansa SA, Barker FK, Heaney LR. 2006. The pattern and timing of diversification of Philippine endemic rodents: evidence from mitochondrial and nuclear gene sequences. Systematic Biology 55:73-88.

Johnson WE. 2010. Endless forms most viral. PLoS Genetics 6:e1001210.

Katoh K, Standley DM. 2014. MAFFT: iterative refinement and additional methods. Springer.

Katzourakis A, Gifford RJ. 2010. Endogenous viral elements in animal genomes. PLoS Genetics 6:e1001191.

381 Katzourakis A, Tristem M, Pybus OG, Gifford RJ. 2007. Discovery and analysis of the first endogenous lentivirus. Proceedings of the National Academy of Sciences 104:6261-6265

383 Kuhn JH, Becker S, Ebihara H, Geisbert TW, Johnson KM, Kawaoka Y, Lipkin WI, 384 Negredo AI, Netesov SV, Nichol ST. 2010. Proposal for a revised taxonomy of the 
family Filoviridae: classification, names of taxa and viruses, and virus abbreviations. Archives of Virology 155:2083-2103.

387 Lanfear R, Calcott B, Ho SY, Guindon S. 2012. PartitionFinder: combined selection of

Omilian AR, Taylor DJ. 2001. Rate acceleration and long-branch attraction in a conserved gene

\section{Neumann K, Michaux J, Lebedev V, Yigit N, Colak E, Ivanova N, Poltoraus A, Surov A,} Markov G, Maak S. 2006. Molecular phylogeny of the Cricetinae subfamily based on the mitochondrial cytochrome $b$ and $12 \mathrm{~S}$ rRNA genes and the nuclear vWF gene. Molecular phylogenetics and evolution 39:135-148. of cryptic daphniid (Crustacea) species. Molecular Biology and Evolution 18:2201-2212. 
411 Patel MR, Emerman M, Malik HS. 2011. Paleovirology--ghosts and gifts of viruses past.

412 Current Opinion in Virology 1:304-309.

413 Pearson W. 2004. Finding protein and nucleotide similarities with FASTA. Current Protocols in 414 Bioinformatics:3-9.

415 Praetorius C, Grill C, Stacey SN, Metcalf AM, Gorkin DU, Robinson KC, Van Otterloo E, 416 Kim RS, Bergsteinsdottir K, Ogmundsdottir MH. 2013. A polymorphism in IRF4 417 affects human pigmentation through a tyrosinase-dependent MITF/TFAP2A pathway. $418 \quad$ Cell 155:1022-1033.

419 Rambaut A. 2012. FigTree v1. 4. University of Edinburgh, Edinburgh, UK Available at: $420 \quad$ http://tree bio ed ac uk/software/figtree.

421 Ronquist F, Teslenko M, van der Mark P, Ayres DL, Darling A, Höhna S, Larget B, Liu L, 422 Suchard MA, Huelsenbeck JP. 2012. MrBayes 3.2: efficient Bayesian phylogenetic inference and model choice across a large model space. Systematic Biology 61:539-542.

Sanderson MJ, Wojciechowski MF, Hu J-M, Khan TS, Brady SG. 2000. Error, bias, and long-branch attraction in data for two chloroplast photosystem genes in seed plants. Molecular Biology and Evolution 17:782-797.

Sharp PM, Simmonds P. 2011. Evaluating the evidence for virus/host co-evolution. Current Opinion in Virology 1:436-441.

Steppan SJ, Adkins RM, Anderson J. 2004. Phylogeny and divergence-date estimates of rapid radiations in muroid rodents based on multiple nuclear genes. Systematic Biology 53:533553.

432 Suzuki Y, Gojobori T. 1997. The origin and evolution of Ebola and Marburg viruses. Molecular Biology and Evolution 14:800-806.

434 Tanne E, Sela I. 2005. Occurrence of a DNA sequence of a non-retro RNA virus in a host plant 
genome and its expression: evidence for recombination between viral and host RNAs. Virology 332:614-622.

437 Taylor DJ, Ballinger MJ, Bowman SM, Bruenn JA. 2013. Virus-host co-evolution under a 438 modified nuclear genetic code. PeerJ 1:e50.

439 Taylor DJ, Bruenn J. 2009. The evolution of novel fungal genes from non-retroviral RNA $440 \quad$ viruses. BMC Biology 7:88.

441 Taylor DJ, Dittmar K, Ballinger MJ, Bruenn JA. 2011. Evolutionary maintenance of filovirid$442 \quad$ like genes in bat genomes. BMC Evolutionary Biology 11:336.

443 Taylor DJ, Leach RW, Bruenn J. 2010. Filovirids are ancient and integrated into mammalian $444 \quad$ genomes. BMC Evolutionary Biology 10:193.

445 Taylor DJ, Piel WH. 2004. An assessment of accuracy, error, and conflict with support values 446 from genome-scale phylogenetic data. Molecular Biology and Evolution 21:1534-1537.

447 Verstrepen L, Verhelst K, Carpentier I, Beyaert R. 2011. TAX1BP1, a ubiquitin-binding adaptor protein in innate immunity and beyond. Trends in Biochemical Sciences 36:347354.

Wahl-Jensen V, Bollinger L, Safronetz D, de Kok-Mercado F, Scott DP, Ebihara H. 2012.

451 Use of the Syrian Hamster as a New Model of Ebola Virus Disease and Other Viral 452 Hemorrhagic Fevers. Viruses 4:3754-3784.

453 Wertheim JO, Kosakovsky Pond SL. 2011. Purifying selection can obscure the ancient age of 454 viral lineages. Molecular Biology and Evolution 28:3355-65. 


\section{Figure 1}

Phylogenetic relationships of filovirid NP-like paleoviruses in mammalian genomes and amino acid sequences from extant filovirids.

Bayesian posterior probabilities for the extant filovirus clade greater than 0.95 are shown as black circles. The phylogeny is based on an alignment with transitive consistency scores $<3$ filtered. The Blue colors represent branches leading to rodent sequences. Red colors represent branches leading to extant viral sequences. Black bars represent branches leading to non-rodent mammalian sequences. Taxonomic labels indicate phylogenetic placement of sequences from specimens assigned to the given taxon. 


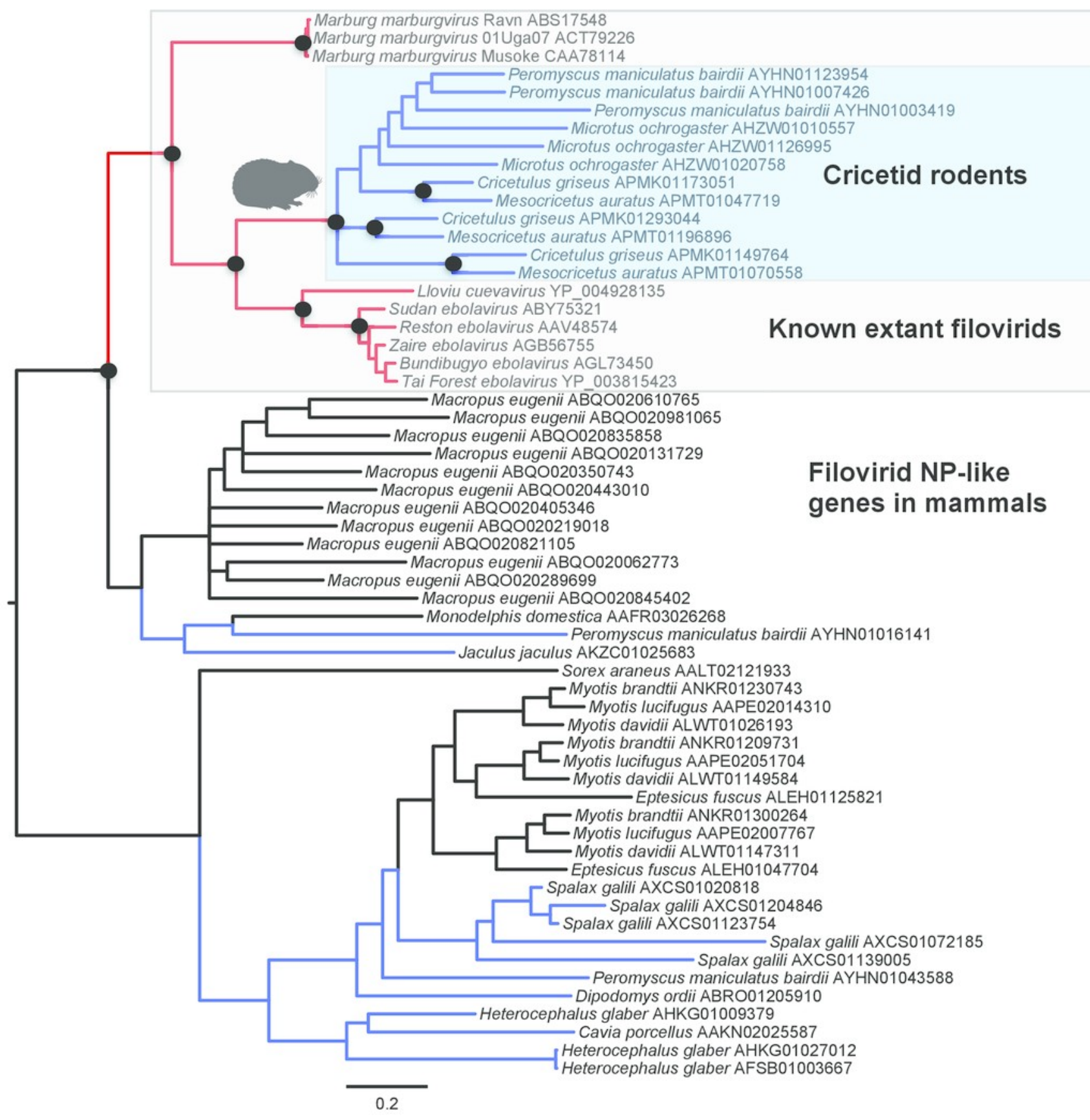




\section{Figure 2}

Phylogenetic relationships of filovirid VP35-like paleoviruses in mammalian genomes and amino acid sequences from extant filovirids.

Bayesian posterior probabilities for the extant filovirus clade greater than 0.95 are shown as black circles. The phylogeny is based on an alignment with transitive consistency scores $<3$ filtered. Blue colors represent branches leading to rodent sequences. Red colors represent branches leading to extant viral sequences. Black bars represent branches leading to nonrodent mammalian sequences. Taxonomic labels indicate phylogenetic placement of sequences from specimens assigned to the given taxon. 


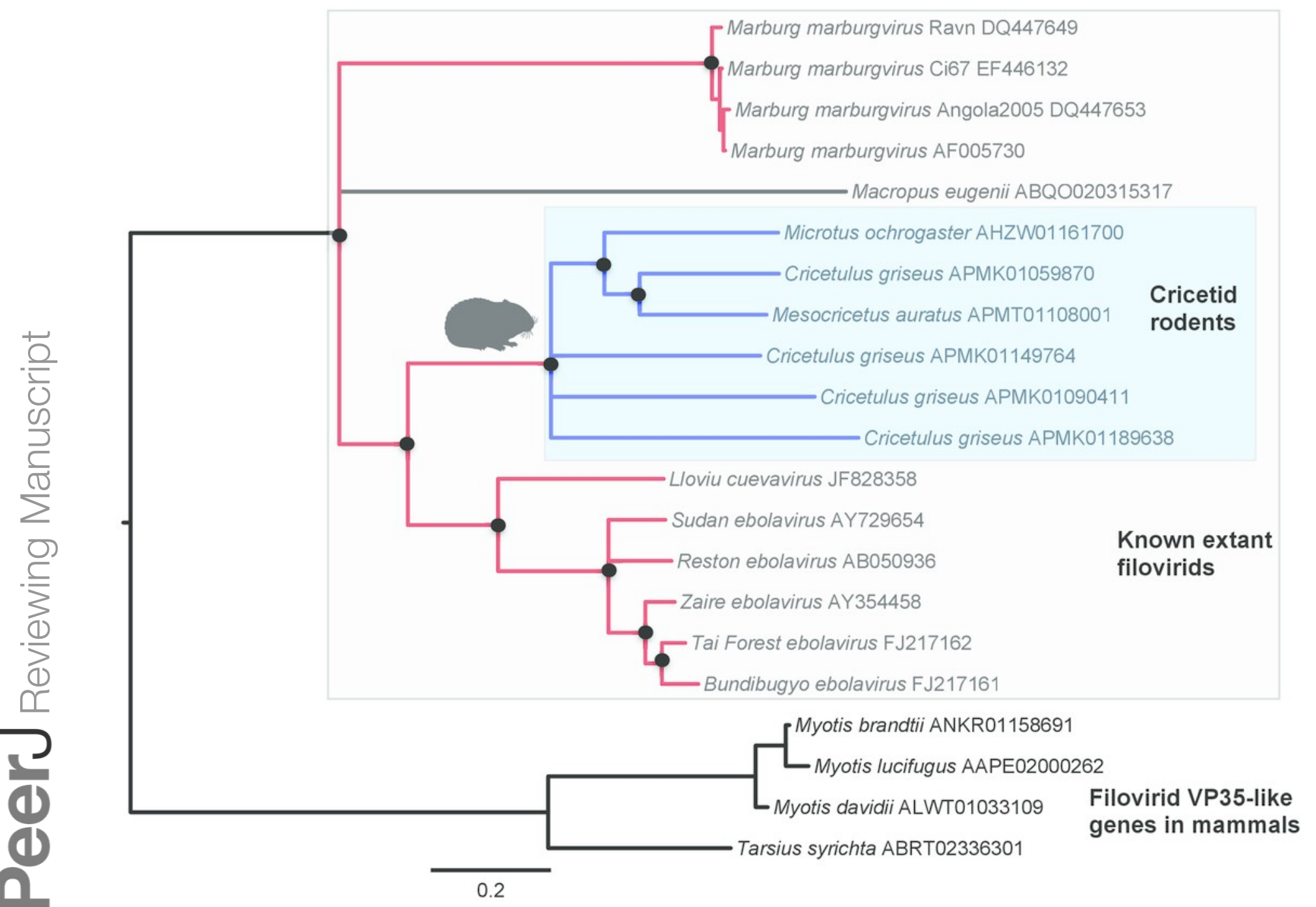




\section{Figure 3}

DNA sequence validation of integration for the filoviral VP35-like sequence in voles of the genus Microtus.

The section of the intron common to rodent introns is highlighted in gray and the proposed filovirid-like insert is highlighted in red. Sequence comparisons (colored blocks are differences) between the PCR product (black bar) for the meadow vole (M. pennsylvanicus) and the genome assembly for the prairie vole (M. ochrogaster) are shown for $A$. the shared intron of rodent genomes and B. the proposed insert containing a filovirid VP35-like sequence in genomes of cricetid rodents.

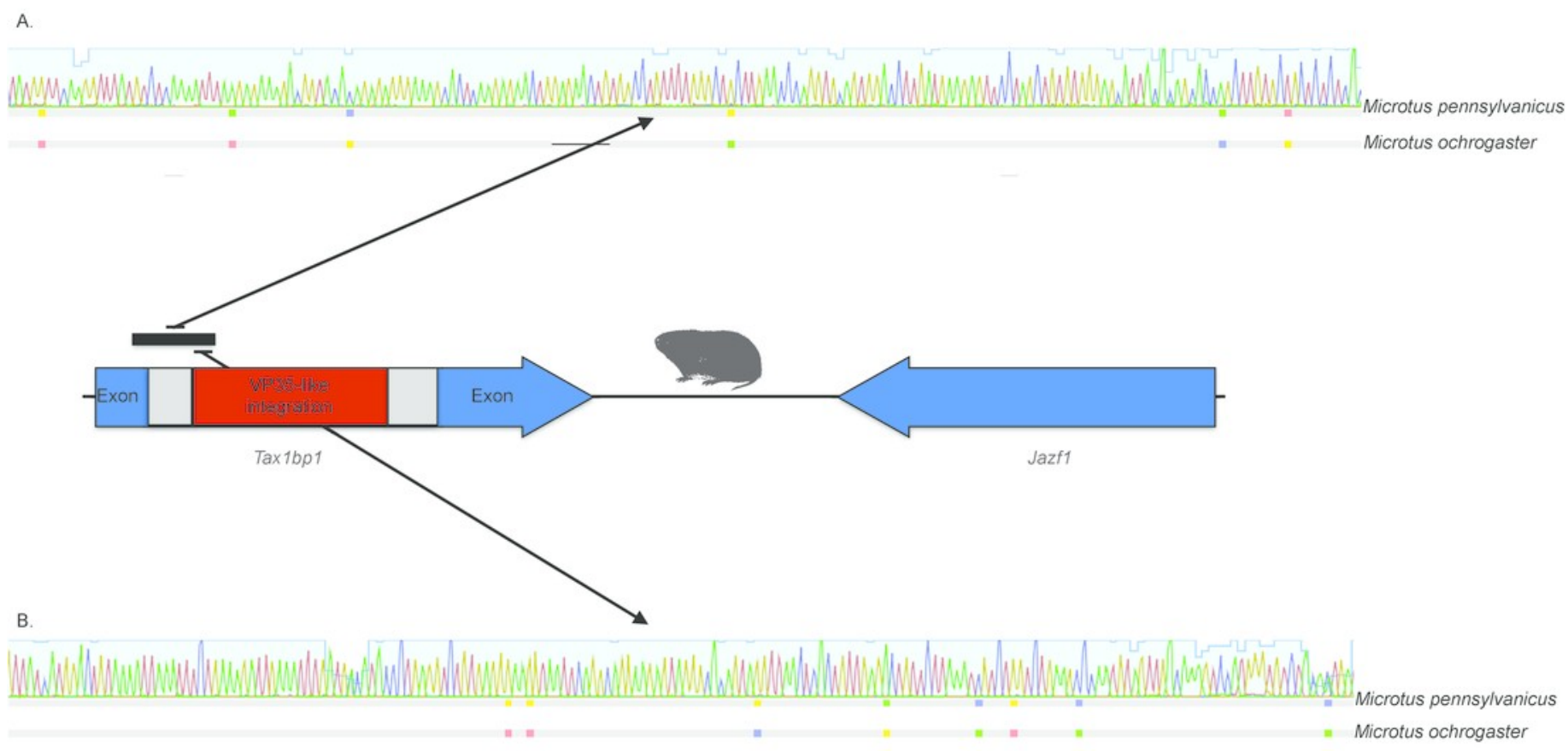




\section{Figure 4}

Graphs of phylogenetic support values for the branch that groups rodent sequences with ebolaviruses and cuevaviruses to the exclusion of marburgviruses

A. the NP-like region and $\mathrm{B}$. the VP35-like region. The $\mathrm{x}$-axis represents the size of the alignment after culling sites according to their transitive consistency scores (TCS). Note that successive removal of the sites that most disagree among pairwise alignments fails to erode support for the branch in question until the alignment size is small. aLRT's are approximate likelihood ratio tests.

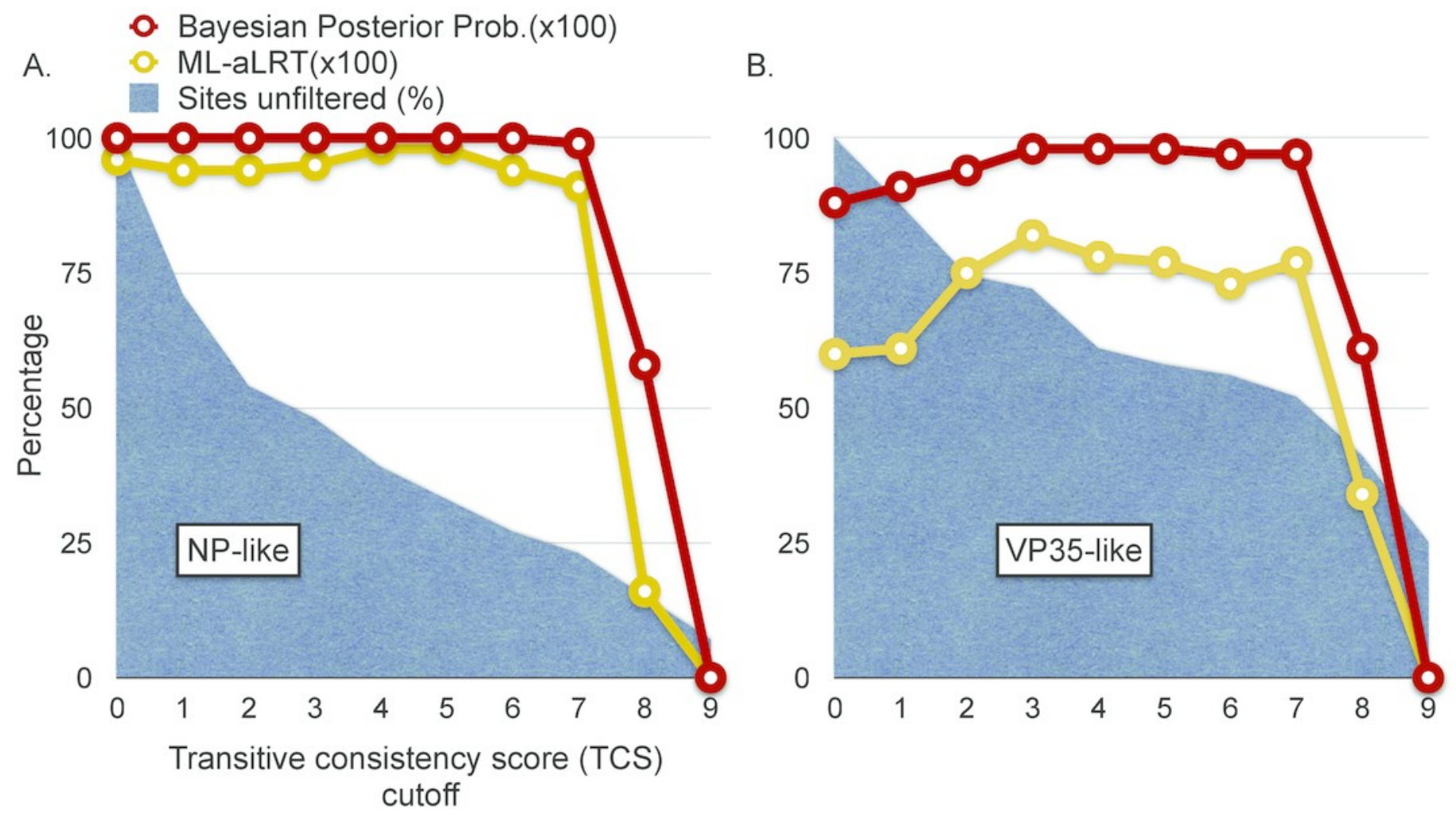




\section{Figure 5}

Cartoons comparing an orthologous genomic region among rodents that $A$. lack a filovirid VP35-like insert; and B. possess an orthologous filovirid VP35-like insert.

Closeups of the upstream and downstream putative insertion boundaries are shown revealing microsynteny at the nucleotide level (the blocks of differing colors are nucleotides). A gray bar represents intronic sequence and a red bar represents the putative filovirid VP35-like insert region.

A.

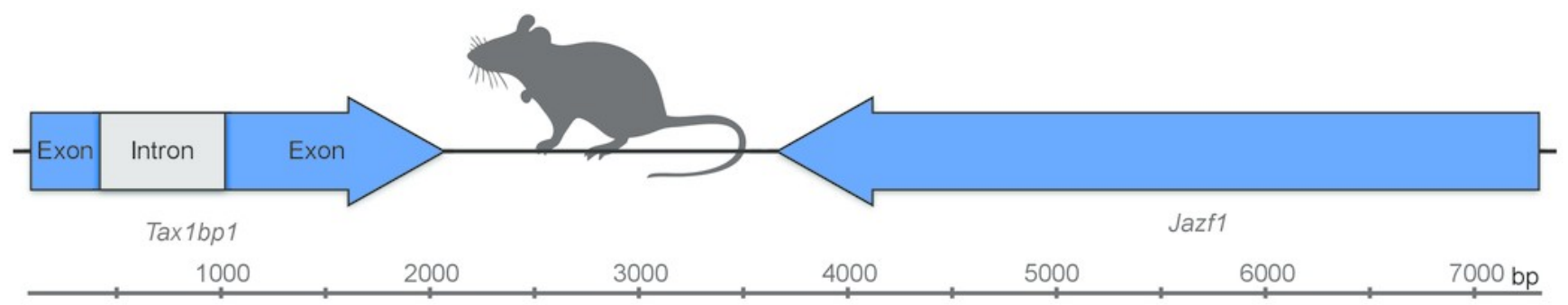

B.

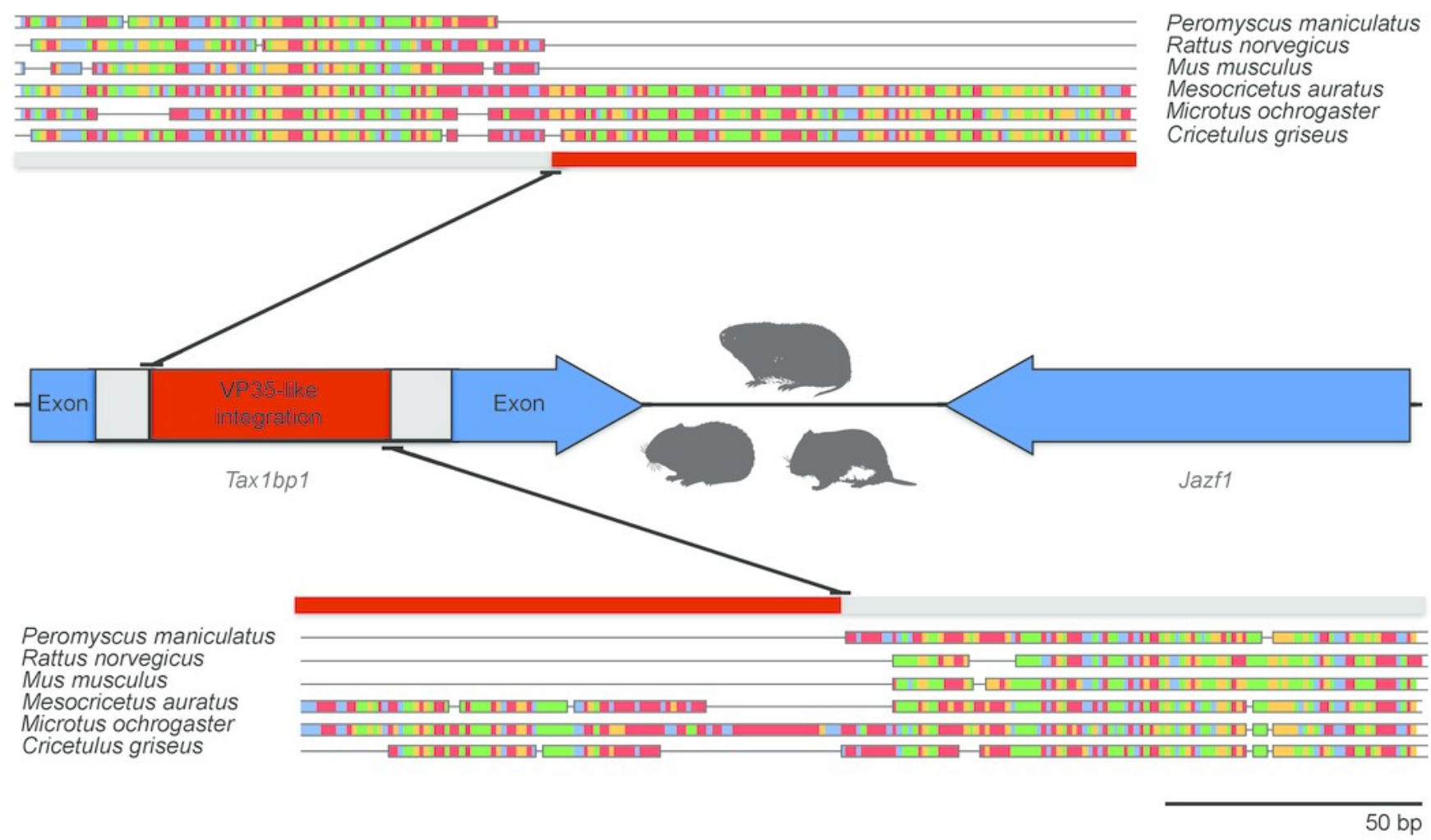




\section{Figure 6}

Cartoon comparing the aligned genomic regions of cricetid rodents that contain a putative orthologous filovirid NP-like sequence.

For this genomic segment, the assembly from the North American deermouse (Peromyscus manipulates bairdi) lacks a detectable insert. Specimens of Microtus ochrogaster, Cricetulus griseus, and Mesocricetus auratus share an insert in the same intergenic region. However, the insert of the prairie vole (M. ochrogaster) is about $10 \mathrm{kbp}$ upstream of the shared insert of members of the other species. The identity bar reveals strong sequence similarity in this intergenic region and flanking the insert site of the striped dwarf hamster (C. griseus) and the golden hamster (M. auratus). Most non-identity is due to indels in the intergenic region.

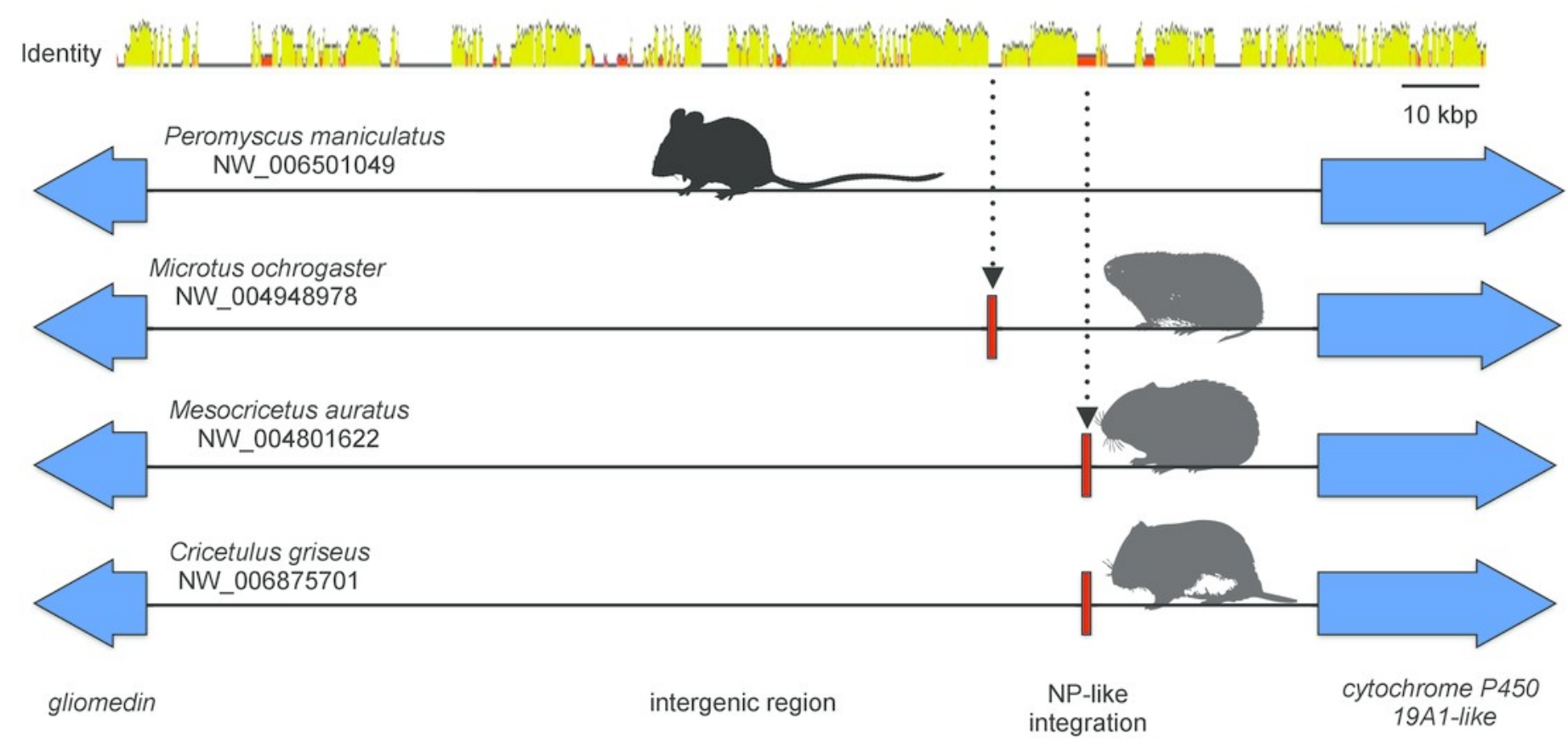




\section{Figure 7}

A graph comparing genetic distance (patristic) among putatively orthologous filovirid-like gene inserts in cricetid rodent genomes.

The left panel compares pairwise distances for the VP35-like inserts with those based on the intronic background. The right panel compares distances of the NP-like inserts with those of their intergenic sequence background.
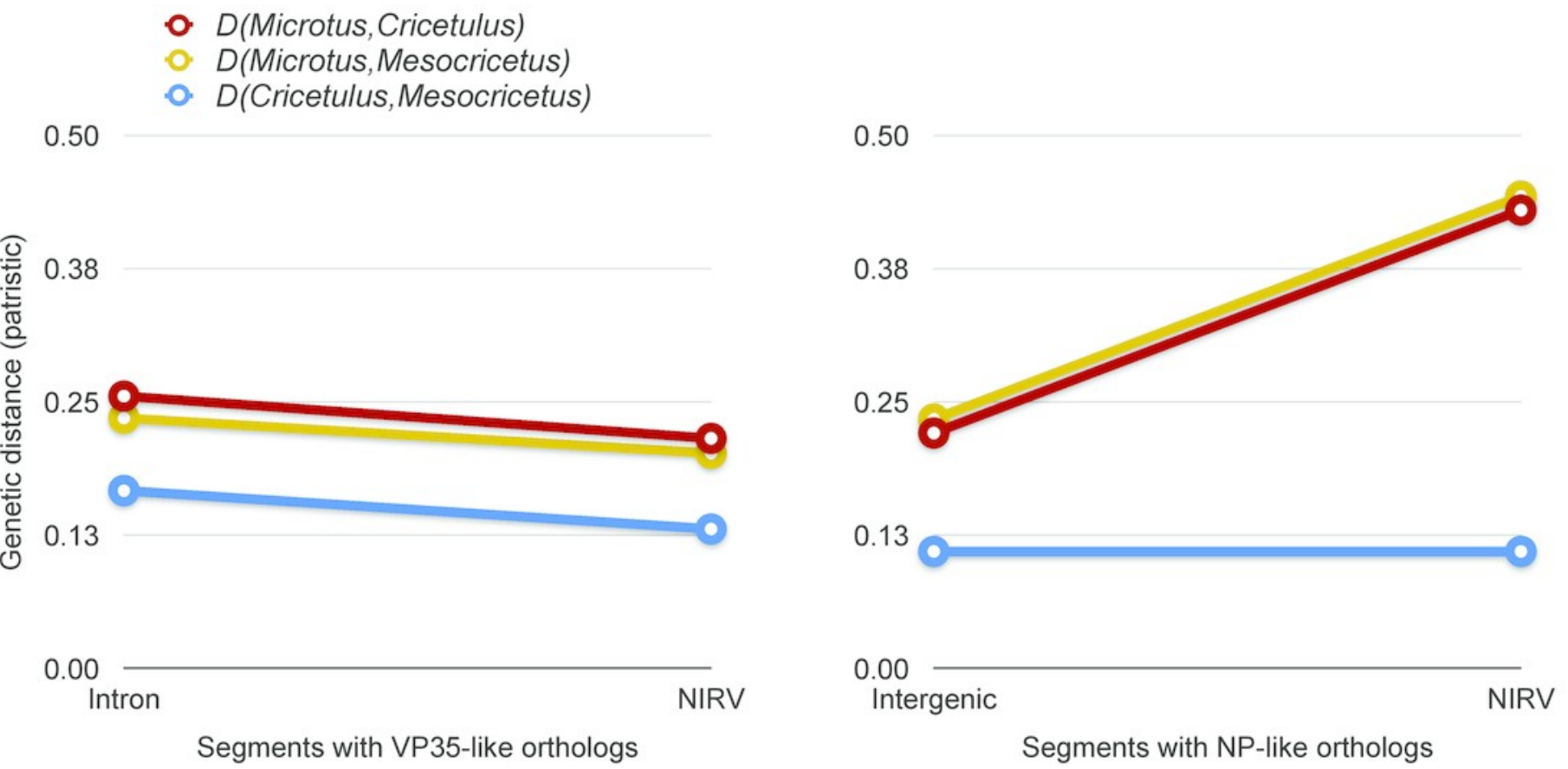


\section{Table $\mathbf{1}_{\text {(on next page) }}$}

Exploration of rooting, taxon set, and filtering of rapidly evolving sites on the phylogenetic position of filovirid-like endogenous genes in cricetid rodents.

Likelihood scores and aLRT branch support values are given for the best topologies found. Observed ingroup topologies are shown in Newick format (brackets and commas) where $\mathrm{E}$ stands for ebolaviruses, L for Lloviu virus, $\mathrm{C}$ for cricetid filovirid-like and $\mathrm{M}$ for marburgvirus sequences. 


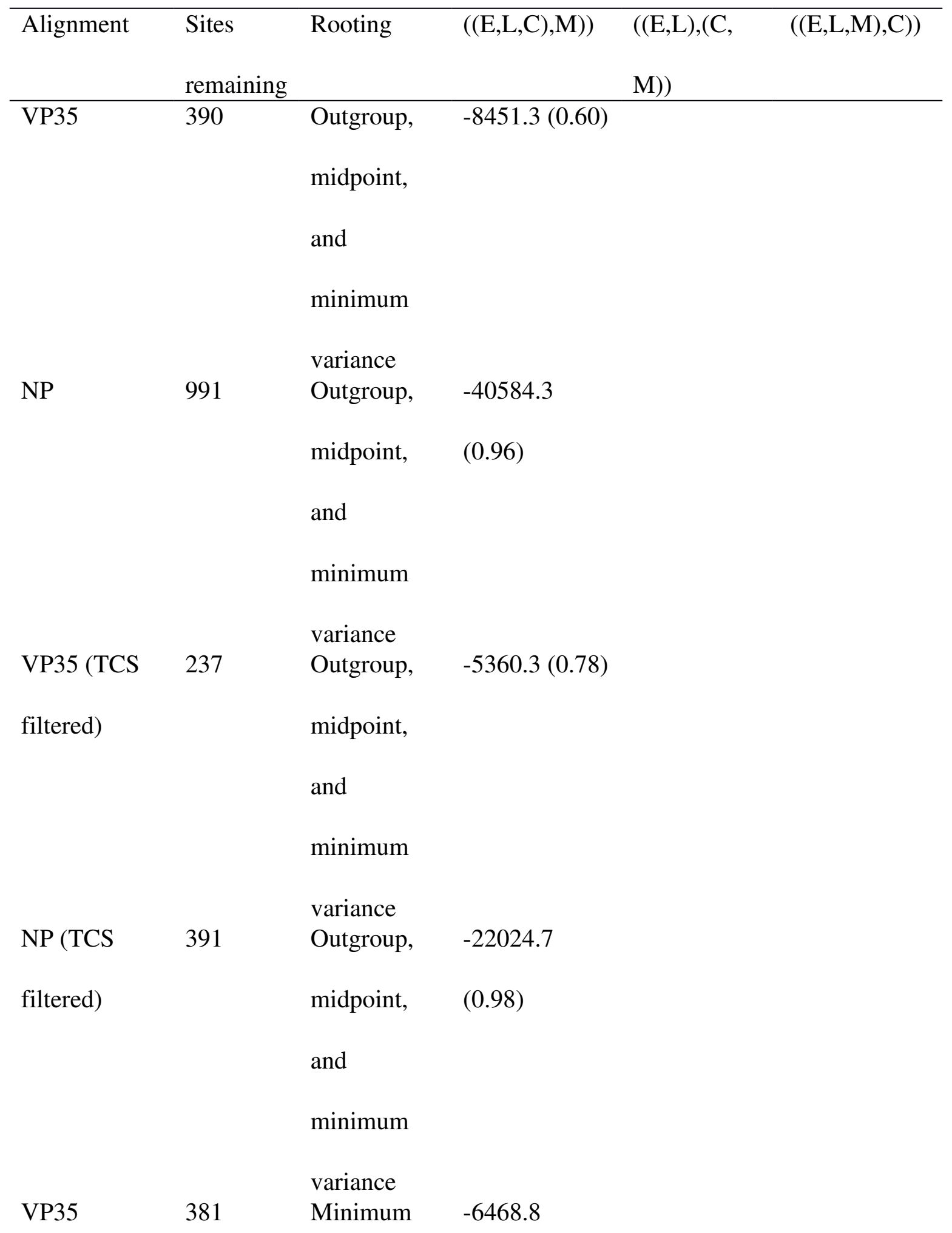




\begin{tabular}{|c|c|c|c|c|c|}
\hline $\begin{array}{l}\text { ingroup } \\
\text { VP35 }\end{array}$ & 280 & $\begin{array}{l}\text { variance } \\
\text { Minimum }\end{array}$ & $\begin{array}{l}(1.0) \\
-5061.6\end{array}$ & & \\
\hline ingroup (TCS & & variance & $(0.96)$ & & \\
\hline \multicolumn{6}{|l|}{ filtered) } \\
\hline NP ingroup & 858 & $\begin{array}{l}\text { Minimum } \\
\text { variance }\end{array}$ & & & $-19899.3(1.0)$ \\
\hline $\begin{array}{l}\text { NP ingroup } \\
\text { (TCS }\end{array}$ & 487 & $\begin{array}{l}\text { Minimum } \\
\text { variance }\end{array}$ & & & $-10813.9(1.0)$ \\
\hline \multicolumn{6}{|l|}{ filtered) } \\
\hline ingroup & & variance & & $(0.99)$ & \\
\hline \multicolumn{6}{|l|}{ ancestral } \\
\hline \multicolumn{6}{|l|}{ NIRV } \\
\hline NP ingroup & 858 & Minimum & & -10834.9 & \\
\hline ancestral & & variance & & $(1.0)$ & \\
\hline \multicolumn{6}{|l|}{ NIRV } \\
\hline ingroup & & variance & & & \\
\hline \multicolumn{6}{|l|}{ ancestral } \\
\hline \multicolumn{6}{|l|}{ NIRV (rapid } \\
\hline \multicolumn{6}{|l|}{ sites filtered) } \\
\hline NP ingroup & 534 & Minimum & $-5692.5(1.0)$ & & \\
\hline ancestral & & variance & & & \\
\hline \multicolumn{6}{|l|}{ NIRV(rapid } \\
\hline sites filtered) & & & & & \\
\hline
\end{tabular}




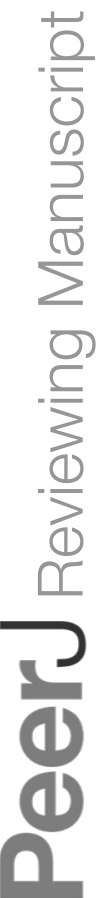

PeerJ reviewing PDF | (v2014:07:2379:1:0:NEW 12 Aug 2014) 